\title{
A Si Optical Modulator based on Fano-like Resonance
}

\author{
H. Du, W. Zhang, W. Cao, D. J. Thomson, C. G. Littlejohns, X. Yan, D. T. Tran, M. Banakar, G. T. \\ Reed
}

\begin{abstract}
In this paper, we present an experimental demonstration of a $\mathrm{Si}$ optical modulator based on Fano-like resonance, which is the first of its kind in the literature. The Fanolike resonance is obtained through weak coupling between two optical resonators in the prototype device and a $P N$ junction is designed for carrier-depletion type electro-refractive modulation. $20 \mathrm{~Gb} / \mathrm{s}$ data rate on-off keying $(\mathrm{OOK})$ is obtained experimentally on the prototype device. Compared with a ring resonator modulator with a similar optical resonance linewidth, a Fano-like resonance based modulator can potentially have a higher extinction ratio (ER) because the Fano-like resonance has an asymmetric spectrum line shape and the line shape on one side can be sharper than the Lorentz resonance line shape of a ring resonator.
\end{abstract}

Index Terms-Si optical modulator, Fano-like resonance, carrier depletion modulation

\section{INTRODUCTION}

$\mathrm{S}$ ILICON photonics is being widely studied for interchip links because of its capability of higher data rates, smaller device footprints, simpler circuits, and capability for multiplexing, when compared with electrical links [1-4]. At the same time, $\mathrm{Si}$ photonics is CMOS-compatible and thus $\mathrm{Si}$ photonic devices can be manufactured with high yield at low cost. Si optical modulators are key Si photonic components to realize chip-to-chip interconnects with the merits mentioned above. A main-stream class of the $\mathrm{Si}$ optical modulator is the Mach-Zehnder interferometer (MZI) modulator, which is relatively tolerant to fabrication tolerance and temperature change $[1,5,6]$. However, an MZI modulator usually consumes higher power, has a larger footprint and a lower ER with a given driving voltage [7]. Ring resonator modulators are another popular class of $\mathrm{Si}$ optical modulators, which can have much smaller footprints but higher modulation efficiency because ring resonator modulators use optical resonance, which is more sensitive to phase change [8-12]. The spectrum line shape of the resonance of a ring resonator is usually Lorentzian and symmetric. To further improve the modulation efficiency of a resonance-based modulator, one can consider manipulating the

This paragraph of the first footnote will contain the date on which you submitted your paper for review. It will also contain support information, including sponsor and financial support acknowledgment. For example, "This work was supported in part by the U.S. Department of Commerce under Grant BS123456."

All the authors are with Zepler Institute for Photonics and Nanoelectronics, University of Southampton, Southampton SO17 1BJ, UK (e-mail: H.Du@ soton.ac.uk). line shape of the resonance to make it asymmetric and sharper on one side of the resonance. The use of Fano-like resonance can achieve this. Comparing the line shape of Fano-like resonance, which is asymmetric, and the line shape of ring resonator resonance, which is symmetric, when these two types of resonance have similar linewidths, the line shape of the Fanolike resonance on one side can be sharper than the line shape of the ring resonator resonance. In theory, the ER of the Fano-like resonance can be higher in this case. Furthermore, it has been proposed to adopt Fano-like resonance for modulation with higher linearity $[13,14]$. However, no high-speed modulator using Fano-like resonance has been experimentally demonstrated in the literature.

In this paper, we design a weakly coupled optical resonators (which are Fabry-Perot (FP) cavities) system with Fano-like resonance. The PN junction is designed for carrier-depletion type electro-refractive modulation, which is popular for its relatively high speed $[7,15,16]$. Yet, a carrier-depletion modulator usually has relatively low modulation efficiency. However, using the resonance based modulation mechanism, the weakness of modulation efficiency of the carrier-depletion modulator can be compensated. The upper boundary of the modulation bandwidth of a resonance based carrier-depletion modulator is mainly limited by the resonance linewidth [17, 18]. In this case and given the discussion above, a Fano-like resonance based modulator can potentially have a higher ER than a ring resonator modulator with a similar resonance linewidth when they are working at the same modulation speed. Additionally, the length of the PN junction of the prototype device is $200 \mu \mathrm{m}$, which is much shorter than the ones in typical MZI modulators and the whole device footprint is just $100 \mu \mathrm{m}$ by $560 \mu \mathrm{m}$. In other words, the Fano-like resonance modulator proposed in this paper has the merit of small footprint, similar to that of ring resonator modulators, whilst the modulation efficiency can potentially be even higher as discussed above. In experiments, with a voltage swing of $2 \mathrm{~V}, 20 \mathrm{~Gb} / \mathrm{s}$ data rate OOK modulation with an ER of $2.76 \mathrm{~dB}$ and $10 \mathrm{~Gb} / \mathrm{s}$ data rate OOK modulation with an ER of $3.42 \mathrm{~dB}$ are demonstrated.

D. J. Thomson acknowledges funding from the Royal Society for his University Research Fellowship.

All data supporting this study are available upon request from the University of Southampton repository at https://doi.org/10.5258/SOTON/D1843.

Copyright (c) 2019 IEEE. Personal use of this material is permitted. However, permission to use this material for any other purposes must be obtained from the IEEE by sending a request to pubs-permissions@ieee.org. 
(a)

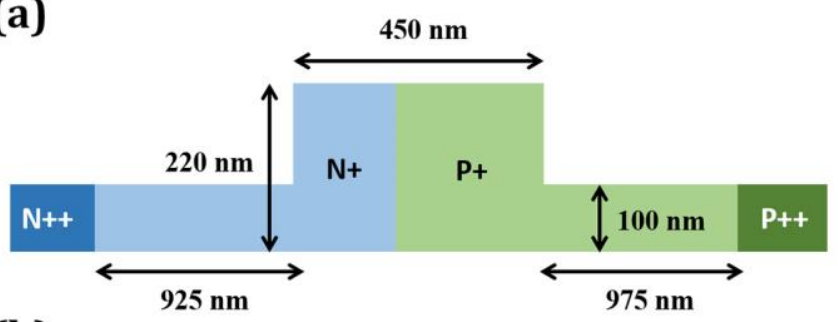

(b)

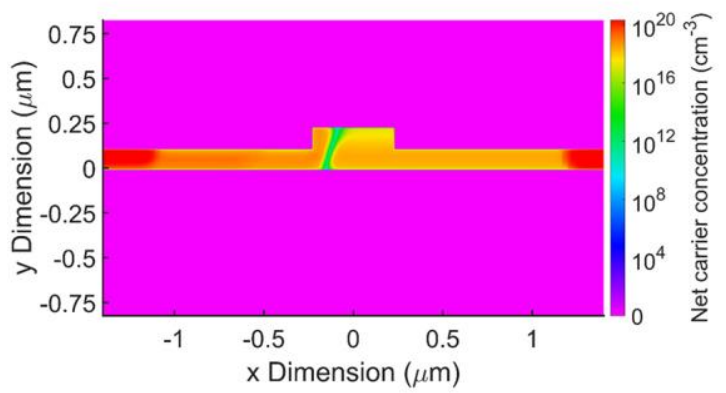

(c)

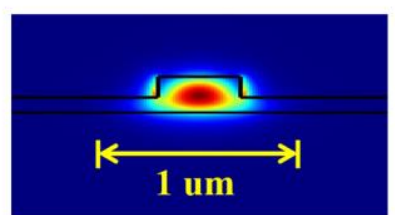

Fig. 1. (a) schematic of the PN junction design on the optical waveguide. (b) Simulated doping profile on the optical waveguide with the PN junction. (c) Simulated optical mode profile of the optical waveguide.

\section{DESIGN}

A prototype device is fabricated through our CORNERSTONE multi-project wafer (MPW) service [19, 20] and the design of it is as follows. The cross section view of the waveguide with the PN junction is given in Fig. 1(a) and the key dimensions are annotated. The length of the PN junction phase shifter is just $200 \mu \mathrm{m}$. As can be seen, the highly-doped regions $(\mathrm{P}++$ and $\mathrm{N}++)$ are moderately distanced from the waveguide, which is a compromise to the trade-off between the modulation bandwidth, ER and the device insertion loss. If the highly-doped regions are closer to the waveguide, the speed of the modulator can be higher. The reason lies in the fact that by putting highly-doped regions closer to the waveguide, the resonance linewidth is increased because the absorption loss is increased, and at the same time the resistance of the PN junction is reduced, both of which can contribute to a wider modulation bandwidth [17, 18]. However, the higher absorption loss can result in higher insertion loss of the device and a less sharp spectrum line shape of the Fano-like resonance, which leads to lower modulation efficiency. Consequently, the design as shown in Fig. 1(a) is adopted. The simulated doping profile of the waveguide cross section is given in Fig. 1(b). As can be seen, there is a distance of about $0.9-1 \mu \mathrm{m}$ between the waveguide edge and the regions with intensive dopants. Fig. 1(c) shows the optical mode profile of the fundamental transverse-electric (TE) mode of the waveguide, which is used in the electro-optic modulation. Comparing Fig. 1(b) and 1(c), we can see that there is almost no overlap between the optical (a)

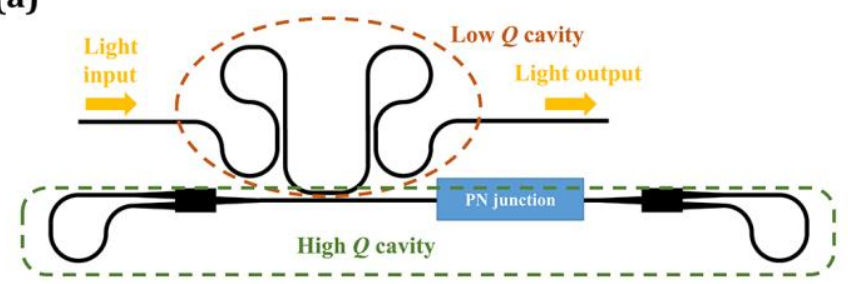

(b)

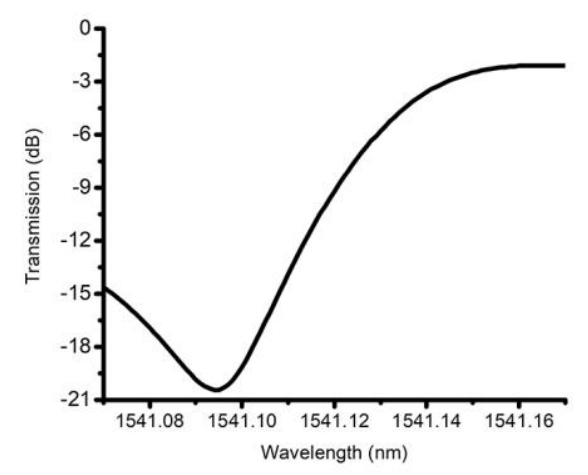

(c)

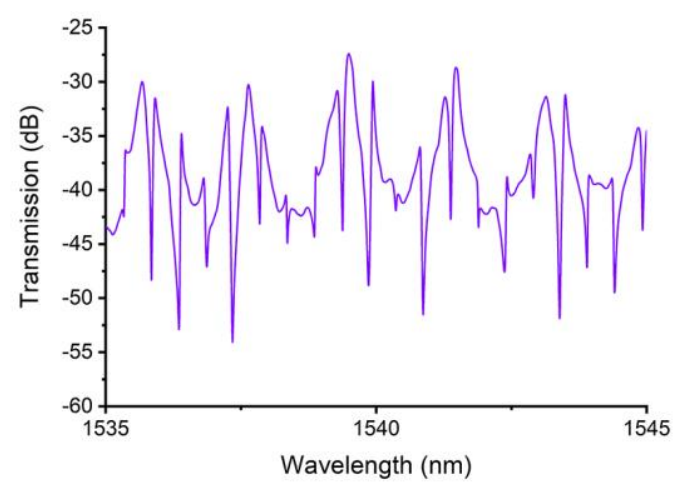

(d)

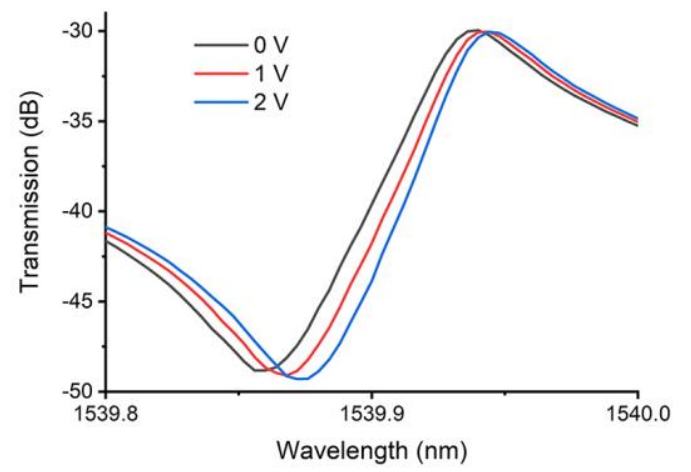

Fig. 2. (a) Schematic of the optical design of the prototype device. (b) Optical spectrum of the device obtained from numerical simulation. (c) Experimentally measured optical spectrum of the device. (d) Spectra of the Fano-like resonance near $1539.9 \mathrm{~nm}$ when different reverse bias voltages are applied to the PN junction.

mode and the regions with intensive dopants, which can result in slight absorption loss.

The optical design of the prototype device is schematized in Fig. 2(a). The Fano-like resonance is formed by weakly coupling a high quality factor ( $Q$ factor) cavity and a low $Q$ factor cavity, the mechanism of which has been discussed in a preliminary study [21]. Briefly speaking, there are two Fabry- 
Perot cavities which are evanescently coupled together. The lower one (see Fig. 2(a)) consists of two mirrors, which are formed by a $1 \times 2$ multi-mode interferometer (MMI) and a Sagnac loop respectively and this is a high $Q$ cavity. The upper cavity (see Fig. 2(a)) consists of two partially transmitting elements (PTEs), which are formed by a directional coupler and a Sagnac loop. This is a low $Q$ cavity, the $Q$ factor of which can be manipulated by engineering the reflection coefficient of the PTEs through designing the coupling rate of the directional coupler. The light is coupled in and out through the PTEs. The Fano-like resonance can be obtained when $\mu^{2} \leq \frac{1}{4}\left(\frac{1}{\tau_{1}}-\frac{1}{\tau_{2}}\right)^{2}$ $\frac{1}{4} \Delta \omega^{2}$, where $\mu$ is the coupling coefficient between the two cavities, $\frac{1}{\tau_{1,2}}$ are the total decay rates of the high $Q$ cavity and the low $Q$ cavity respectively, and $\Delta \omega$ denotes the resonance frequency detuning between the two cavities. (See details in [21].)

To realize optical modulation, a PN junction is built in the high $Q$ cavity. By applying a reverse bias voltage to the PN junction, the free carrier density in the optical mode region reduces and thus the effective index of the waveguide is changed due to the plasma depletion effect, and eventually the optical resonance and thus the output intensity of the device is modulated.

The device is designed to have the Fano-like resonance spectrum as plotted in Fig. 2(b), which is the numerical simulation result using the scattering matrix method. This resonance line shape has a peak transmission at about $-2 \mathrm{~dB}$ and the slope is about $0.5 \mathrm{~dB} / \mathrm{pm}$.

\section{EXPERIMENTAL RESULTS}

The optical spectrum of the prototype device is given in Fig. 2(c) where multiple Fano-like resonance line shapes can be identified. We choose the resonance near $1539.9 \mathrm{~nm}$ for the modulation experiments and the wavelength of $1539.918 \mathrm{~nm}$ is selected as the working wavelength. This resonance and the working wavelength is chosen to have a good balance between the transmission and the modulation depth. The measured transmission at the selected working wavelength is about -44 $\mathrm{dB}$, with the loss at the grating couplers included. The total loss at the input and output grating couplers is estimated to be 13.5 $\mathrm{dB}$ using the measurement result from a reference device, whilst the loss at the connections between the grating couplers and the device is negligible. In this case, the insertion loss of the device at the working wavelength is estimated to be 30.5 $\mathrm{dB}$. The peak transmission of the selected Fano-like resonance is measured to be about $-30 \mathrm{~dB}$. Similarly, the insertion loss of the device at the peak wavelength is estimated to be $16.5 \mathrm{~dB}$. In addition, the slope of the resonance line shape is about 0.2 $\mathrm{dB} / \mathrm{pm}$. Compared with the simulated resonance in Fig. 2(b), the resonance performance measured on the prototype device is inferior. The degradation is likely caused by imperfect fabrication, which will be discussed in detail later. The spectra of this resonance with different reverse bias voltages applied to the PN junction are shown in Fig. 2(d). The static ER is about $3.5 \mathrm{~dB}$ between the two states at $0 \mathrm{~V}$ and $2 \mathrm{~V}$ reverse bias. If the resonance can have the performance as designed in Fig. 2(b), the static ER can reach about $9 \mathrm{~dB}$. (a)

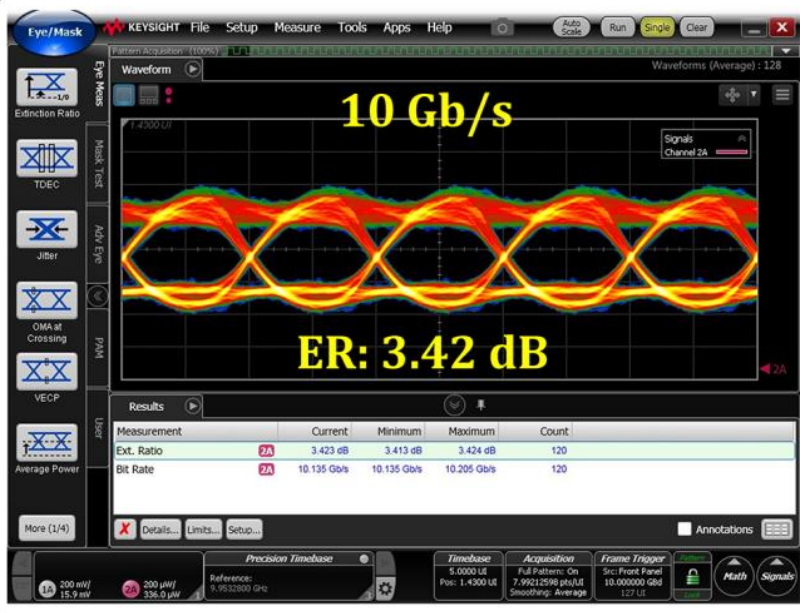

(b)

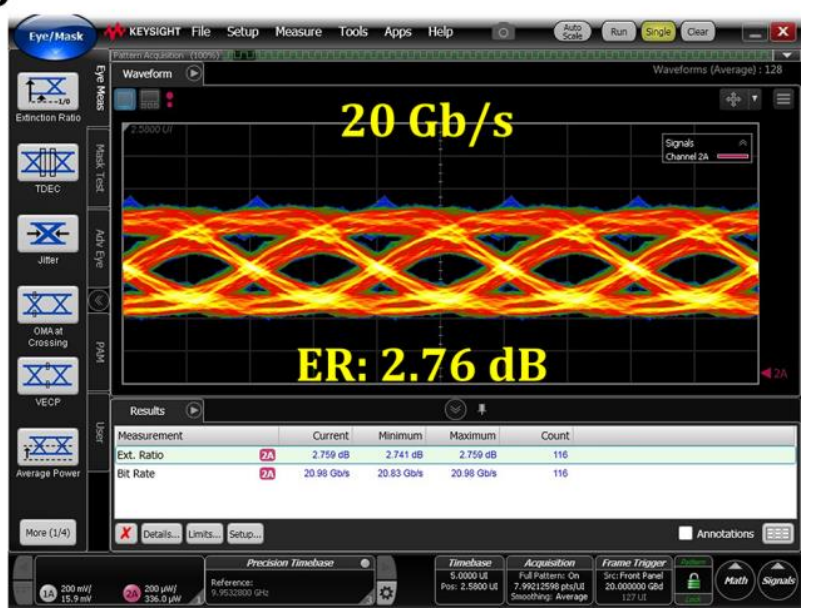

Fig. 3. Eye diagrams at (a) $10 \mathrm{~Gb} / \mathrm{s}$ OOK and (b) $20 \mathrm{~Gb} / \mathrm{s}$ OOK.

In the high-speed modulation experiments, a reverse bias voltage of $1 \mathrm{~V}$ and a pseudo-random bit sequence (generated by SHF 12104A, a bit pattern generator (BPG)) with a singleended voltage swing of $2 \mathrm{~V}$ are applied to the device. Continuous wave (CW) light generated from a tunable laser source at the wavelength of $1539.918 \mathrm{~nm}$ is launched into the device. The modulated optical output from the device is amplified by an erbium-doped fiber amplifier (EDFA) and then collected by a digital communication analyzer (Keysight 86100D). Finally, eye diagrams at $10 \mathrm{~Gb} / \mathrm{s}$ OOK and $20 \mathrm{~Gb} / \mathrm{s}$ OOK are obtained as shown in Fig. 3(a) and 3(b) with ERs of $3.42 \mathrm{~dB}$ and $2.76 \mathrm{~dB}$ respectively.

The performance of the device can be further improved. The low transmission is mainly caused by the mismatch between the designed parameters and the parameters of the fabricated device. To be specific, the transmission of the device is very sensitive to the reflection coefficient of the PTEs in the low $Q$ cavity as verified numerically. The higher the reflection coefficient is, the lower the transmission will be. The PTE reflection coefficient is controlled by the coupling rate of the directional coupler of the PTE, which is very sensitive to the width of the waveguide and the coupling gap. Consequently, the transmission is very sensitive to the dimension tolerance in the fabrication. With better fabrication dimension control, the general transmission of the device can be much improved. 
It is also possible to improve the ER of the device. As measured on the reference devices, the optical loss at the PN junction is about $20 \mathrm{~dB} / \mathrm{cm}$, which is worse than our expectation, whilst in the numerical simulation of Fig. 2(b), the loss at the PN junction is assumed to be about $10 \mathrm{~dB} / \mathrm{cm}$. The loss directly reduces the sharpness of the resonance spectrum line shape and consequently reduces the ER of the electro-optic modulation. If we can reduce the optical loss by refining the fabrication, the device can have much sharper resonance spectrum line shape and thus a higher ER.

\section{CONCLUSION}

In conclusion of this study, we have experimentally investigated the electro-optic modulation of a $\mathrm{Si}$ modulator based on Fano-like resonance. The Fano-like resonance is generated by weakly coupling two FP cavities with large $Q$ factor contrast. The PN junction is designed for carrierdepletion modulation and integrated in the high $Q$ cavity. For characterization of the device performance, optical tests, DC electro-optic tests, and high speed modulation tests are performed. In the high speed electro-optic test, $20 \mathrm{~Gb} / \mathrm{s}$ data rate OOK with an ER of $2.76 \mathrm{~dB}$ and $10 \mathrm{~Gb} / \mathrm{s}$ data rate $\mathrm{OOK}$ with an ER of $3.42 \mathrm{~dB}$ are realized when a driving voltage swing of $2 \mathrm{~V}$ is applied. This study fills the gap of the lack of Si optical modulators based on Fano-like resonance and shows the potentials of such kind of modulators in the situation when short PN junctions but large ERs are required.

\section{REFERENCES}

[1] G. Li et al., "Ring resonator modulators in silicon for interchip photonic links," IEEE Journal of Selected Topics in Quantum Electronics, vol. 19, no. 6, pp. 95-113, 2013.

[2] K. Ohashi et al., "On-chip optical interconnect," Proceedings of the IEEE, vol. 97, no. 7, pp. 1186-1198, 2009.

[3] A. V. Krishnamoorthy et al., "Progress in low-power switched optical interconnects," IEEE Journal of selected topics in quantum electronics, vol. 17, no. 2, pp. 357-376, 2010.

[4] K. Li et al., "Electronic-photonic convergence for silicon photonics transmitters beyond 100 Gbps on-off keying," Optica, vol. 7, no. 11, pp. 1514-1516, 2020.

[5] F. Gardes, D. Thomson, N. Emerson, and G. Reed, "40 Gb/s silicon photonics modulator for TE and TM polarisations," Optics express, vol. 19, no. 12, pp. 11804-11814, 2011.

[6] D. J. Thomson et al., "50-Gb/s silicon optical modulator," IEEE Photonics Technology Letters, vol. 24, no. 4, pp. 234-236, 2011.

[7] A. Rahim et al., "Taking silicon photonics modulators to a higher performance level: state-of-the-art and a review of new technologies," Advanced Photonics, vol. 3, no. 2, p. 024003, 2021.

[8] J.-B. You, M. Park, J.-W. Park, and G. Kim, "12.5 Gbps optical modulation of silicon racetrack resonator based on carrier-depletion in asymmetric pn diode," Optics express, vol. 16, no. 22, pp. 1834018344, 2008.

[9] P. Dong et al., "High-speed and compact silicon modulator based on a racetrack resonator with a $1 \mathrm{~V}$ drive voltage," Optics letters, vol. 35, no. 19, pp. 3246-3248, 2010.

[10] P. Dong et al., "Wavelength-tunable silicon microring modulator," Optics express, vol. 18, no. 11, pp. 10941-10946, 2010.

[11] G. Li et al., "25Gb/s 1V-driving CMOS ring modulator with integrated thermal tuning," Optics Express, vol. 19, no. 21, pp. 20435-20443, 2011.

[12] X. Zheng et al., "Ultralow power $80 \mathrm{~Gb} / \mathrm{s}$ arrayed CMOS silicon photonic transceivers for WDM optical links," Journal of Lightwave Technology, vol. 30, no. 4, pp. 641-650, 2012.
[13] X. Xie, J. Khurgin, J. Kang, and F.-S. Chow, "Linearized machzehnder intensity modulator," IEEE Photonics Technology Letters, vol. 15, no. 4, pp. 531-533, 2003.

[14] S. Chen, G. Zhou, L. Zhou, L. Lu, and J. Chen, "High-linearity Fano resonance modulator using a microring-assisted Mach-Zehnder structure," Journal of Lightwave Technology, vol. 38, no. 13, pp. 3395-3403, 2020.

[15] G. T. Reed, G. Mashanovich, F. Y. Gardes, and D. Thomson, "Silicon optical modulators," Nature photonics, vol. 4, no. 8, pp. 518-526, 2010.

[16] G. T. Reed et al., "Recent breakthroughs in carrier depletion based silicon optical modulators," Nanophotonics, vol. 3, no. 4-5, pp. 229245, 2014.

[17] H. Yu et al., "Trade-off between optical modulation amplitude and modulation bandwidth of silicon micro-ring modulators," Optics express, vol. 22, no. 12, pp. 15178-15189, 2014.

[18] I.-L. Gheorma and R. Osgood, "Fundamental limitations of optical resonator based high-speed EO modulators," IEEE Photonics Technology Letters, vol. 14, no. 6, pp. 795-797, 2002.

[19] http://www.cornerstone.sotonfab.co.uk/.

[20] C. G. Littlejohns et al., "CORNERSTONE's Silicon Photonics Rapid Prototyping Platforms: Current Status and Future Outlook," Applied Sciences, vol. 10, no. 22, p. 8201, 2020.

[21] H. Du et al., "Ultra-sharp asymmetric Fano-like resonance spectrum on Si photonic platform," Optics express, vol. 27, no. 5, pp. 73657372, 2019. 